\section{A study of horse's blood constituents and weight variations during endurance competitions}

\author{
Joana Nery', Anna Assenza², Walter Pinna ${ }^{3}$, Antonio Sfuncia ${ }^{3}$ \\ and Domenico Bergero'
}

Dipartimento di Produzioni Animali, Epidemiologia ed Ecologia, Università di Torino ${ }^{1}$, Dipartimento MOBIFIPA, Università di Messina ${ }^{2}$ and Dipartimento di Biologia Animale, Università di Sassari ${ }^{3}$ (Italy)

\section{Introduction}

Endurance horses have been the target of several exercise physiology studies over the past few years due to the particularly prolonged exercise and physical demand to the animal. Previous studies (Farris et al. 1998, Assenza et al. 2004, Bergero et al. 2005) have focused deeply the variation of blood serum concentration on branched chain amino acids (BCAA), and its' use as energy source, tryptophan, non-esterified fatty acids (NEFA) and serotonin during exercise and its' association with the concept of overall fatigue (Newsholme et al. 1987). Miller-Graber et al. (1991) studies suggested a muscle's amino acid catabolism during exercise due to the observed increase in alanine blood concentration. Haematologically, an increase on haematocrit (packed cell volume, PCV) levels during exercise was reported (Farris et al. 1998) and explained by the mobilization of red blood cells to the blood stream during strenuous exercise (Persson et al. 1973, Pagan 2000). According to Bergero (2004), electrolytes, particularly potassium and chloride, are lost with sweat during exercise while Harris (2000) refers the water loss from sweating as a cause of decrease in the circulatory volume. According to Pagan (2000), in a performance horse nutrition perspective, it is fundamental to consider carefully both energy and electrolytes.

The aim of this study was to set the framework of endurance exercise physiology features during competitions regarding blood concentration variations of several components, weight variation related to water and electrolyte losses and control of the feeding plan in order to understand the extent of its' influence on horses' performance.

\section{Material and methods}

Six endurance competition horses were monitored during 4 competitions. Blood sampling was undertaken before competition, after each $30 \mathrm{~km}$ (until 90), and after competition (1, 6, 12 and 48 hours). Blood analysis considered the blood levels of amino acids (arginine, aspartic acid, methionine, alanine, glycine, isoleucine, lysine, leucine, valine, tyrosine, phenylalanine and tryptophan), serotonin, NEFA, urea, creatinine, total protein, electrolytes (calcium, chloride, potassium, magnesium and sodium) and haema- tological constituents (blood cells, PCV, haemoglobin and platelets).

Weighting was accomplished, in the last two competitions, immediately before and after each $30 \mathrm{~km}$ and one hour after competition. Both blood sampling values and weights were statistically analysed throughout the competition and recovery period. The feeding plan, ingredients and quantities, was monitored between the third and fourth competitions.

\section{Results}

The blood analysis results have highlighted a steady decrease of BCAA and a decrease with no statistical evidence of tryptophan blood serum concentration throughout all competitions. Regarding recovery period both BCAA and tryptophan have shown a tendency to return to the resting values (except leucine). All the other amino acids monitored, except aspartic acid and methionine, have revealed a steady decrease during competition; recovery was characterized by different patterns among amino acids. NEFA blood concentration decreased during competition and a recovery to resting values during the post-competition period was observed. Urea blood concentration has slightly increased during late competition while maintenance of this concentration during recovery was observed. Both creatinine and total protein have shown a slight decrease in the second 30 kilometres competition; during recovery a statistical decrease for total protein was observed. Serotonine blood concentration has shown, despite the statistical evidence, a peak at $60 \mathrm{~km}$ competition and a slight increase during recovery. All electrolyte blood concentrations have shown a significant decrease during competition except sodium and magnesium (the last with no statistical evidence). During recovery all electrolytes' concentration, besides magnesium, differed significantly from the resting value at six hours recovery (higher for sodium and lower for chloride, calcium and potassium). Blood cell analysis did not show many significant variations throughout competition and recovery (monitored for the first hour) except regarding leukocytes and erythrocytes. Erythrocytes, PCV and haemoglobin have shown to increase slightly during competition (with no statistical evidence). Leukocytes variation was associated to the statistical difference found in the percentage of granulocytes that has increased significantly between 30 and $60 \mathrm{~km}$ competition reaching a statistically different percentage from the resting value.

Weight variation and electrolyte loss were also monitored: at a 60-kilometer competition there is a statistically significant impact on horse's weight (from $417.44 \pm 27.19 \mathrm{~kg}$ to $379.00 \pm 15.56 \mathrm{~kg}$ ) and that recovery's first hour is the period in which weight values return to resting values. Dehydration extent values ranged between $4.88 \%$ and $11.84 \%$ and sodium, potassium and chloride observed average losses were $52.00 \pm 16.55 \mathrm{~g}, 37.79 \pm 12.03 \mathrm{~g}$ and $128.80 \pm 41.00 \mathrm{~g}$ respectively.

From the feeding plan analysis it was possible to observe a slightly lower supply of energy, an evident higher supply of protein, a modest higher supply of electrolytes and a marked lower supply of sodium compared to the horses' requirements, estimated according to the French system. 


\section{Discussion}

Blood analysis on endurance competition horses pointed out an utilization of BCAA as an energy source for exercise as supported by the references. On the other hand, tryptophan's decrease until the 6th hour recovery is questionable due to the lack of statistical evidence. According to the references, the concept of overall fatigue is associated with the levels mentioned together with NEFA and serotonine blood concentration but since an increase in tryptophan and NEFA was not verified and the peak on serotonine blood level has shown no statistical evidence it is possible to infer from the results that the horses did not reach the mentioned status. The decrease on arginine, lysine, alanine, glycine, tyrosine and phenylalanine blood concentrations suggest an utilization of these amino acids during competition. It was suggested previously by different authors that there is a muscle's amino acid catabolism during exercise which is a possible explanation to the mentioned amino acids increase in the blood stream.

Based on the results and references it is possible to infer an utilization of the mentioned amino acids as a source of energy which is accomplished mainly in the liver, where occurs a degradation of amino acids in order to provide carbon skeletons for ATP, glucose or fat production and an excretion of nitrogen as ammonia. On the other hand, urea blood concentration results did not show evidences of high amino acid degradation which indicates that amino acids were mobilised in the blood stream but its' catabolism in the liver was not determinant to increase urea level in the blood. The decrease on electrolytes blood concentration is associated to the sweat loss, due to its' composition, and as supported by the calculation preformed based on the weight loss during competition. Sodium blood concentration increase might be related to the increase of blood cells (particularly erythrocytes) in the blood stream in order to maintain the osmotic pressure. Regarding leukocytes and considering that exercise was the varying factor it is difficult to explain an immunologic response during exercise. Red blood cells results pointed out a mobilization to the blood stream as widely defended by the references consulted. Regarding weight loss, since no water and feed was administered after each $30 \mathrm{~km}$ competition, it is possible to deduce that the cause relies on variations occurring during exercise particularly due to sweating.

The results contribute to explain that a 60-kilometre competition has an important impact on horse's weight and that the first hour post-competition is essential for horse's weight recovery. Considering results obtained on weight variation it is possible to calculate a $5.22 \%$ body weight water loss for the competitions studied, which is translated in a circulatory volume decrease and consequently influences indirectly the increase on blood cell parameters mentioned.

At a nutritional level energy slight deficiency does not constitute a bottleneck to performance achievement but the observed excessive protein supply might have deleterious consequences regarding water requirements and "accumulation of nitrogen end-products". Electrolytes' nutritional balance was not problematic except for sodium and considering its' losses in sweat it would be important to reach an adequate supply of this element in the diet. No changes were undertaken between nutritional analyses in both controlled competitions.

\section{Conclusions}

Regarding blood analysis it is possible to conclude that there was an important mobilization of amino acids in the blood stream during competition which should be related to its' use as energy source. It is also possible to conclude that the horses did not reach a status of fatigue during competition due its' NEFA and serotonine blood levels. It is particularly important to consider the weight losses that occur during competition and are of most importance in horse management. The specific long-lasting exercise must be carefully controlled in order to maintain a good condition and performance of competing horses. At a nutritional level it would be advisable to consider the discussion points mentioned in order to fulfil the horses' needs mostly regarding protein excess and electrolytes deficiency. In a general approach it would be important that competing horses' diet is formulated according to their specific needs and take a special attention to protein and electrolyte levels.

\section{References}

Assenza A., Bergero D., Tarantola M., Piccione G. and Caola G. (2004): Blood serum branched chain amino acids and tryptophan modifications in horses competing in long-distance rides of different length. Journal of Animal Physiology and Animal Nutrition 88, 172-177

Bergero D. (2004): New insights related to the nutritional management of endurance horses. Proceedings of the 2nd European Equine Health and Nutrition Congress, Lelystad, Nederlands, 41-50

Bergero D., Assenza A. and Caola G. (2005): Contribution to our knowledge of the physiology and metabolism of endurance horses. Livestock Production Science 92, 167-176

Danielsen K., Lawrence L., Siciliano P. and Thompson K. (2000): Effect of diet on weight loss and plasma variables in endurance exercised horses. In: Pagan J. D. (editor): Advances in Equine Nutrition. Kentucky Equine Research Inc. Nottingham University Press, 297-298

Farris J. W., Hinchcliff K. W., McKeever K. H., Lamb D. R. and Thompson D. L. (1998): Effect of tryptophan and of glucose on exercise capacity of horses. Journal of Applied Physiology 85, 807-816

Harris P. (2000): Tied up with electrolytes - or not? In: Pagan J. D. (editor). Advances in Equine Nutrition. Kentucky Equine Research Inc. Nottingham University Press, 513-530

Miller-Graber P. A., Lawrence L.M., Kurcz E., Bump K., Kane R. and Fisher M. (1991): Dietary protein level and energy utilization during submaximal treadmill exercise in the horse. Journal of Nutrition 121, 1462-1469

Newsholme E. A., Acworth I. N. and Blomstrand E. (1987): Amino acids, brain neurotransmitters and a functional link between muscle and brain that is important in sustained exercise. In: Benzi G. (editor): Advances in Myochemistry. London: John Libby Eurotext, 127-138

Pagan J. D. (2000): Choke points: what factors limit performance in the equine athlete? In: Pagan J. D. (editor): Advances in Equine Nutrition. Kentucky Equine Research Inc. Nottingham University Press, 125-28

Persson S. G. B. and Lydin G. (1973): Circulatory effects of splenectomy in the horse: III. Effect on pulse-work relationship. Zentralblatt für Veterinärmedizin A20, 521
J. Nery
Dipartimento di Produzioni Animali, Epidemiologia ed Ecologia Università di Torino
Italy
neryjoana@hotmail.com 